\title{
Texture Comparison with an Orientation Matching Scheme
}

\author{
Nguyen Cao Truong Hai*, Do-Yeon Kim and Hyuk-Ro Park*
}

\begin{abstract}
Texture is an important visual feature for image analysis. Many approaches have been proposed to model and analyze texture features. Although these approaches significantly contribute to various image-based applications, most of these methods are sensitive to the changes in the scale and orientation of the texture pattern. Because textures vary in scale and orientations frequently, this easily leads to pattern mismatching if the features are compared to each other without considering the scale and/or orientation of textures. This paper suggests an Orientation Matching Scheme (OMS) to ease the problem of mismatching rotated patterns. In OMS, a pair of texture features will be compared to each other at various orientations to identify the best matched direction for comparison. A database including rotated texture images was generated for experiments. A synthetic retrieving experiment was conducted on the generated database to examine the performance of the proposed scheme. We also applied OMS to the similarity computation in a K-means clustering algorithm. The purpose of using K-means is to examine the scheme exhaustively in unpromising conditions, where initialized seeds are randomly selected and algorithms work heuristically. Results from both types of experiments show that the proposed OMS can help improve the performance when dealing with rotated patterns.
\end{abstract}

Keywords-Orientation Matching, Texture Analysis, Texture Comparison, K-means Clustering

\section{INTRODUCTION}

Along with color, texture is another important visual attribute used to describe characteristics of regions, or even images. If color gives the general view about the image, texture gives the detail information and characteristics of the content inside. Therefore, texture is an indispensable feature for image analysis and pattern recognition, especially in the areas of content-based image retrieval and object recognition. In addition, there are still a wide range of applications requiring texture information such as geological images, medical image-based (pre) diagnosis, industrial product quality control, etc. This also may explain why texture analysis has drawn a lot of attention from scientists along with a wide variety of different proposed research projects over the years. These approaches can be put into categories such as: statistical analysis and prediction of the pixels (gray-level co-occurrence matrices (GLCM) [1], Markov random field [2]), complex geometric distribution of pixels (fractal dimension [3]), spectral analysis (Gabor filter [4], Fou-

Manuscript received September 2, 2011; first revision May 7, 2012; accepted May 23, 2012.

Corresponding Author: Hyuk-Ro Park

* School of Electronics and Computer Engineering, Chonnam National University, Gwangju, Korea (nct.hai@ejnu.net, doyeon@ejnu.net,hyukro@jnu.ac.kr) 
rier descriptors [5]), and the most recent work is known as the deterministic tourist walk (DTW) algorithm [6]. Although contributing significantly to various image-based applications, most of these methods are, however, sensitive to the changes of scale and orientation of the texture pattern. Unfortunately, these changes are pretty common in many application areas. Recognizing this fact, many scale-invariant and orientation-invariant feature approaches have been suggested such as: locally invariant [7], rotation-invariant and scale-invariant Gabor features [8], etc. Except [8], most other approaches focus on just one property of a texture problem (scale or orientation). [8] tries to solve both problems, but it has a serious drawback in that the rotation-invariant feature and scale-invariant feature cannot be considered at the same time. In an attempt to make the situation easier, we suggest the Orientation Matching Scheme (OMS), which is also capable for scale matching. Although it is easy to apply for any multi-orientation feature extraction approaches, we find it most appropriate to work on the Gabor features. The Gabor features will not only be extracted along with various orientations but they will also be compared to each other at various orientations. The purpose is to reduce the difference between rotated versions of the same kind of texture pattern.

The rest of paper is organized as follows: Section 2 presents the motivation of our work along with the proposed Orientation Matching Scheme description. A modified version of the $K$ means algorithm is also suggested in this section. Section 3 will deliver the settings and result analysis of our experiments. This section also describes a challenge generated texture database, which is derived from the Brodatz dataset. In Section 4, we conclude and give some suggestions about future work.

\section{Orientation Matching Scheme}

\subsection{Motivation}

Gabor filters and wavelet descriptors have suggested efficient ways of crossing scales and orientations to model texture. However, it seems that there is not enough information to directly use the features extracted from these models to compare texture. Although the features are extracted from the texture image at different sequential scales and orientations, there is the great likelihood that they are not being compared at the same scales and orientations. According to the results, a lot of researches on scale-invariant and rotation-invariant texture features have been proposed. In an attempt to solve the problem and preserve the good characteristics of the Gabor filter in texture analyzation, this paper suggests the Orientation Matching Scheme, which can be also extended for scale matching.

The choice of Gabor filters in this work is motivated by the following reasons: the study in [9] shows that Gabor filters have been the best system that matches human vision, and the Gabor filters model provides texture information at any scale and orientation (while the wavelets model provides only three orientations: horizontal, vertical and diagonal orientation). The later reason is more important for our orientation matching.

With the OMS the feature that is extracted from the texture image across scales along various orientations, is also compared with different orientations. Instead of complex and computational invariant features, OMS proposes a simpler way for analyzing texture information via identifying the best-matching orientation between texture samples. OMS also provides an enhancement for current texture analyzing methods, which have problems with rotated textures. 


\subsection{Gabor Feature \& Limitations}

Gabor functions are Gaussian modulated by complex sinusoids (Fig. 1). 2-D Gabor functions can be expressed as [10]:

$$
g(x, y)=\frac{1}{2 \pi \sigma_{x} \sigma_{y}} \exp \left[-\frac{1}{2}\left(\frac{x^{2}}{\sigma_{x}^{2}}+\frac{y^{2}}{\sigma_{y}^{2}}\right)+2 \pi j W x\right]
$$

The Gabor filter masks can be considered as orientation and scale tunable edges and line detectors. The statistics of these micro features in a given region can be used to characterize the underlying texture information. A class of such self-similar functions referred to as Gabor wavelets is discussed in [11]. This self-similar filter dictionary can be obtained by appropriate dilations and rotations of $g(x, y)$ through the generating function:

$$
g_{m n}(x, y)=a^{-m} g\left(x^{\prime}, y^{\prime}\right)
$$

where $x^{\prime}=a^{-m}\left(x \cos \theta_{n}+y \sin \theta_{\mathrm{n}}\right), y^{\prime}=a^{-m}\left(-x \sin \theta_{n}+y \cos \theta_{n}\right), \theta_{n}=n \pi / R, n=0,1, \ldots,(R-1), m=0$, $1, \ldots,(S-1) . R$ is the number of orientations, and $S$ is the number of scales in the multiresolution decomposition, and a $=\left(U_{h} / U_{l}\right)^{1 /(S-1)}$ with $U_{l}$ and $U_{h}$ as the lower and the upper center frequencies of interest [11]. Then, a texture region can be described by a mean and a deviation of the distribution of the transform coefficients.

Assume there is an image $I(x, y)$. The energy distribution of the transformation $J_{m n}$, mean values $\mu_{m n}$ and deviation $\sigma_{m n}$ can then be determined as follows:

$$
\begin{gathered}
J_{m n}(x, y)=\sum_{x_{I}} \sum_{y_{I}} I\left(x_{I}, y_{I}\right) g_{m n}\left(x-x_{I}, y-y_{I}\right) \\
\mu_{m n}=\frac{1}{N} \sum_{x} \sum_{y}\left|J_{m n}(x, y)\right|
\end{gathered}
$$

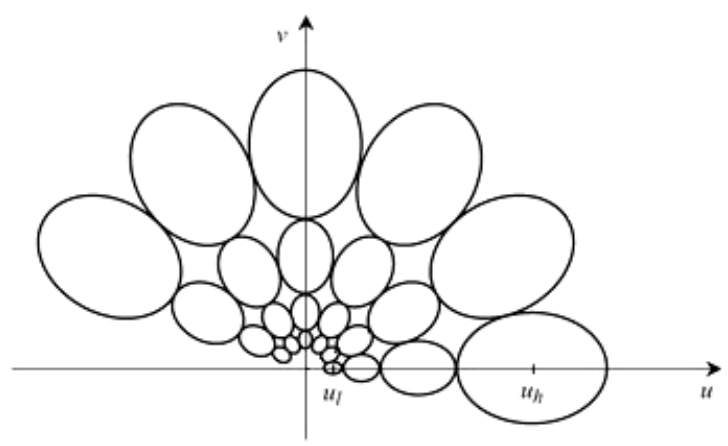

Fig. 1. Gabor filter spectrum: the contours indicate the half-peak magnitude of the filter responses in the Gabor filter dictionary. The filter parameters used are: $U_{h}=0.4, U_{l}=0.05, S=4$ and $R=$ $6[11]$ 


$$
\sigma_{m n}=\sqrt{\frac{1}{N} \sum_{x} \sum_{y}\left(\left|J_{m n}(x, y)\right|-\mu_{m n}\right)^{2}}
$$

Now, a feature vector characterizing the texture region can be constructed using mean values $\mu_{m n}$ and deviation $\sigma_{m n}$ values.

$$
\bar{f}^{G}=\left[\begin{array}{llllll}
\mu_{00} & \sigma_{00} & \mu_{01} & \cdots & \mu_{(S-1)(R-1)} & \sigma_{(S-1)(R-1)}
\end{array}\right]^{T}
$$

Gabor features are then directly compared to each other for recognizing the similar texture regions. It is not difficult to observe that the attributes will be compared at the same scales along the same orientations. This certainly may lead to mismatching two patterns of the same texture that have different orientations in two images. The reason is obvious since the energy distribution of the transformation of each region will be very different at different orientations. This is also why we need to extract texture information at various orientations (and scales), and use those as the presenting feature. So, there is a drawback of directly comparing two Gabor features without considering the orientation of the texture in each region before extracting them from images.

\subsection{OMS Description}

Assume that we use the Gabor feature on the $S$ scales along the $R$ orientations to characterize a texture region. The main idea of the Orientation Matching Scheme is to compare the Gabor features by looking at different orientations. Instead of only comparing two features at the same scale $D$ ( $D$ versus $D$ ), we also perform a pairwise comparison of those vectors at the following different orientations: $D$ versus $(D+\varphi) . \varphi$ is a rotating index within each scale of the Gabor feature information. $\varphi$ is a natural number, and $0<\varphi \leq \mathrm{R}$. The minimum difference between those comparisons is assumed to be the actual distance between two features.

At a specified scale, texture information is extracted from different $R$ orientations. To compare the textures at different orientations, we need to rotate the texture information orders within each scale of feature vector. (Note that we are considering orientation matching problem only.) The rotating index $\varphi$ indicates what the angle is used. This rotation can be expressed by a rearrangement of texture information along orientations inside a texture feature. And for each comparison, we will select two of these rotated feature vectors as candidates. Fig. 2 shows how the scheme works. Assume that $\bar{f}_{0}^{G}$ is the original Gabor feature vector $\left(\bar{f}^{G}\right)$. The rotated vector candidates $\bar{f}_{\varphi}^{G}$ are then identified as follows:

$$
\bar{f}_{\varphi}^{G}=\left[\begin{array}{lllllllll}
\mu_{0 \varphi} & \sigma_{0 \varphi} & \mu_{0(\varphi+1)} & \sigma_{0(\varphi+1)} \ldots & \mu_{k[(j+\varphi) \bmod R]} & \sigma_{k[(j+\varphi) \bmod R]} & \ldots & \mu_{(S-1)(\varphi-1)} & \sigma_{(S-1)(\varphi-1)}
\end{array}\right]^{T}
$$

Let $\bar{f}_{\varphi_{A}}^{G}$ and $\bar{f}_{\varphi_{B}}^{G}$ be the feature vectors of two patterns of comparison. Then, the actual distance $d_{A B}$ between two patterns can be defined as the minimum difference from among the rotated candidates as follows: 


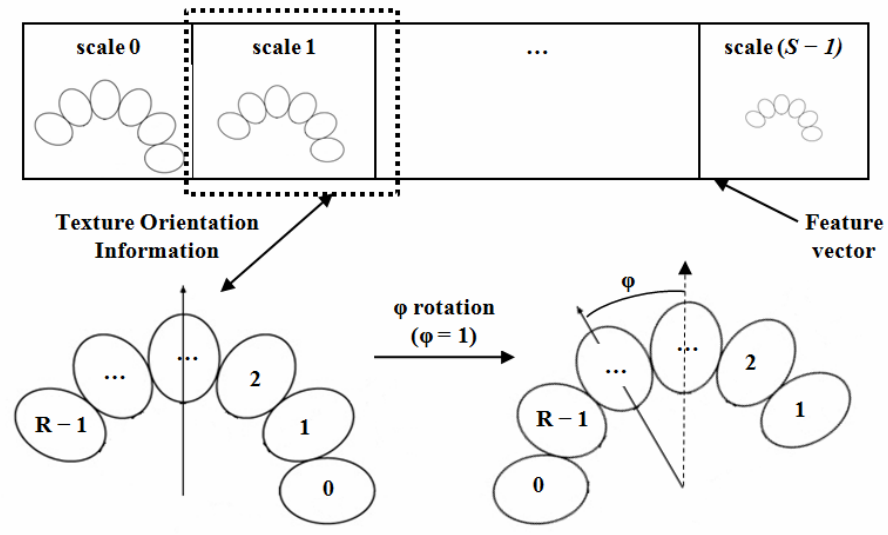

Fig. 2. Description of the Orientation Matching Scheme

$$
d_{A B}=\arg \min _{0<\theta \leq R}\left(\sum_{k=0}^{S-1} \sum_{j=0}^{R-1}\left(\left(\mu_{k[(j+\theta) \bmod R]}^{\varphi_{B}}-\mu_{k j}^{\varphi_{A}}\right)^{2}+\left(\sigma_{k[(j+\theta) \bmod R]}^{\varphi_{B}}-\sigma_{k j}^{\varphi_{A}}\right)^{2}\right)\right)
$$

or

$$
d_{A B}=d_{B A}=\arg \min _{0<\omega \leq R}\left(\sum_{k=0}^{S-1} \sum_{j=0}^{R-1}\left(\left(\mu_{k[(j+\omega) \bmod R]}^{\varphi_{A}}-\mu_{k j}^{\varphi_{B}}\right)^{2}+\left(\sigma_{k[(j+\omega) \bmod R]}^{\varphi_{A}}-\sigma_{k j}^{\varphi_{A}}\right)^{2}\right)\right)
$$

\subsection{K-Means Clustering Using the Orientation Matching Scheme}

$K$-means clustering is applied to the database with scheme enhancement to evaluate the scheme performance. We use $K$-means to exhaustively examine the scheme in unpromising conditions. It is known that the $K$-means works heuristically with random initialized seeds. And during the progress, the $K$-means algorithm does a large number of instance comparisons using OMS. That means the matching scheme is tested repeatedly all the time. In this kind of strict testing environment, we expect that the ability of the proposed scheme will be recognized.

Given a set of observed patterns $\left\{x_{1}, x_{2}, \ldots, x_{N}\right\}$, the $K$-means clustering algorithm aims to partition the $N$ patterns into $K$ subsets $\left\{C_{l}, C_{2}, \ldots, C_{K}\right\}$. Each pattern in the observed set is assigned to a cluster (set) based on a similarity measurement. To ensure a high correlation of members in each set, the $K$-means algorithm tries to minimize the within-set variances turn by turn. The target function for this task can be presented as:

$$
\arg \min _{C_{1}, C_{2}, \ldots, C_{K}} \sum_{i=1}^{K} \sum_{x_{j} \in C_{i}}\left\|x_{j}-\mu_{i}\right\|^{2}
$$

where $\mu_{i}$ is the temporary center pattern of the $i$ th cluster $C_{i}$, and $\left\|x_{j}-\mu_{i}\right\|$ is the Euclidean distance between pattern $x_{j}$ and center $\mu_{i}$ of the $i$ th cluster.

The modified version of $K$-means in this work uses the OMS instead of the direct Euclidean distance for the similarity measurement. That means that the distance between pattern $x_{j}$ and center $\mu_{i}$ is determined by looking at many orientations. The target function under new condi- 
tions can be presented as:

$$
\arg \min _{C_{1}, C_{2}, \ldots, C_{K}} \sum_{i=1}^{K} \sum_{x_{j} \in C_{i}} \arg \min _{\varphi_{1}, \varphi_{2}}\left\|x_{j}^{\left(\varphi_{1}\right)}-\mu_{i}^{\left(\varphi_{2}\right)}\right\|^{2}
$$

where $\varphi_{1}, \varphi_{2}$ are orientation indices. However, there is a chance that the $K$-means cannot decide the optimal solution (looped). The troublemaker is the generated temporary center patterns. This is because they are the results of averaging all members in each cluster and their values are changed over time. This causes a pattern to belong to some clusters along a orientation and may be assigned to another cluster at another orientation. Consequently, a new center pattern is generated for the new state of the cluster. But the pattern, which has just been assigned to this cluster, is somehow assigned vice versa in the new state. So, to prevent the loop, we suggest limiting the number of epochs where the $K$-means can be applied and so the parameter $E$ is applied for that purpose.

\section{EXPERIMENTAL RESULTS}

\subsection{Experiment Settings}

In order to evaluate the performance of our proposed scheme on texture comparison, we specially designed a various scales' database based on the famous Brodatz texture sets. Selected Brodatz images are shown in Fig. 3. For each of the ten selected original $640 \times 640$ Brodatz images, a set of clockwise rotations is applied with bicubic interpolation and anti-aliasing enhancement. Then, for each rotated image, we cropped eight $320 \times 320$ images containing the full texture information (without blanks) at different locations. By this method we obtained 10 sets of 64 new images having the same texture with different content and orientations. The total of 640 rotated texture images of these 10 sets is ready for experiments. Furthermore, among the ten selected original texture samples there are many very similar pairs or groups, which contain mostly horizontal and vertical lines. This intentional selection gives the evaluation more challenges to deal with. Our purpose is to exhaustively test the scheme performance on this challenge database Fig. 2.

J. Han and K.-K. Ma [8] have observed the facts that the Gabor features with the $S=R=4$ decomposition setting is the most appropriate for texture image retrieval application. This con-
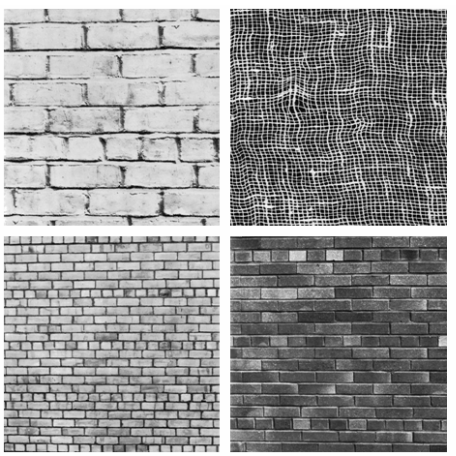
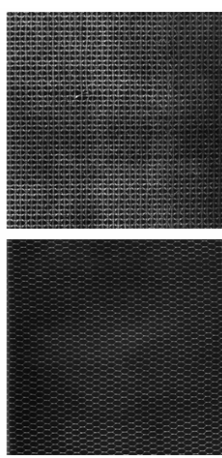
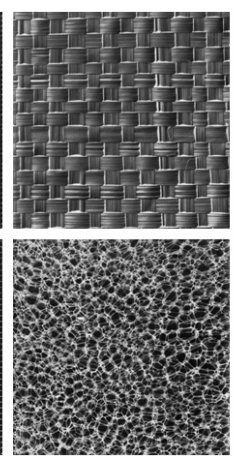

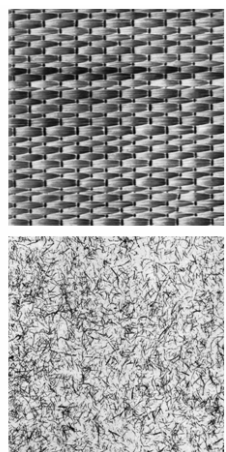

Fig. 3. Ten Brodatz images are manually selected to create testing database 
clusion also implies that the $S=R=4$ decomposition setting is good for texture feature comparison. However, in the case where we need the orientation matching scheme, $R=4$ is too small to be meaningful. Furthermore, in the case of [8] the experiment was conducted with equal sized images. This is a big advantage over our database situation. Moreover, scale-invariant and rotation-invariant features are treated separately and cannot be considered at the same time. These problems will be discussed in another work. In our experiment, $S=3$ and $R=8$ works well. So, according to the scheme mechanism, for each distance measurement we compared features in 8 different orientation situations, and chose the best match result (minimum distance). In order to check the role of the number of epochs, we tested the modified $K$-means algorithm with different numbers of epochs. This number is varied among $E=100,200 \ldots, 1,000,2,000,5,000$, and 10,000 . Actually, the number of epochs is needed just in case the database contains complex textures that are too similar in high scale resolution due to small sizes.

The first experiment would like to test the scheme in unpromising conditions with the $K$ means clustering algorithm. We all know that the $K$-means algorithm depends a lot on the random seeds. To increase the certainty of experiment results, we tried each test several times, and the minimum, maximum, and average clustering performances of each run were recorded. Performance presents the ratio of corrected clustered patterns over the total patterns of the database.

In the second experiment, we randomly chose 20 images from the database ( 2 from each set) to be the query images. We tried to retrieve the top 64 similar images for each query. The precision and recall system was relied upon to assess the retrieval performance. For each query, the precision is the ratio of the total number of ground-truth images from the same set of query images over the $T$ retrieved images. The recall is the ratio of the number of retrieved ground-truth images over the actual number of the ground-truth images from the same set of the query images. After testing each of 20 random images as the query, the trend of the average precision or the average recall can be drawn to evaluate the retrieval performance.

\subsection{Results and Discussion}

Fig. 4 illustrates the first experiment results. Fig. 4 shows that the modified $K$-means, which uses OMS, outperforms the original version. Even in the worst case of the OMS version (the 700 -epoch one), the average minimum performance (68.75\%) is far better than that of the original version $(50.42 \%)$. And the best from our record was $87.50 \%$, which was a $33.75 \%$ improvement over the $53.75 \%$ improvement of the old version. From the result, we can notice that the conventional Gabor feature method does not have the ability to handle the rotated texture recognition problem. Around the 50\% recognition performance means nothing. Instead, with the OMS enhancement the average performance is significantly improved (about 22.88\%). That means the proposed OMS can handle the situation.

The results also indicate that the $K$-means clustering method, which works with random initial seeds, can lead to very surprising results. Even with a very small value for the epoch parameter $E(E \leq 200)$, we can achieve an unexpected significant high of performance. It is easy to guess that we somehow obtained very good initial seeds. This is because the observation in Table 1 implies that the larger number of epochs does not ensure a better clustering result. One clue for that idea has been in recorded in Table 1: when $E=10,000$, the clustering performance is lower than that of $E=2,000$.

The curve of precision of the second experiment is shown in Fig. 5. The observation shows that the trend of the average precision of the OMS looks very stable over that of the queries. And it 


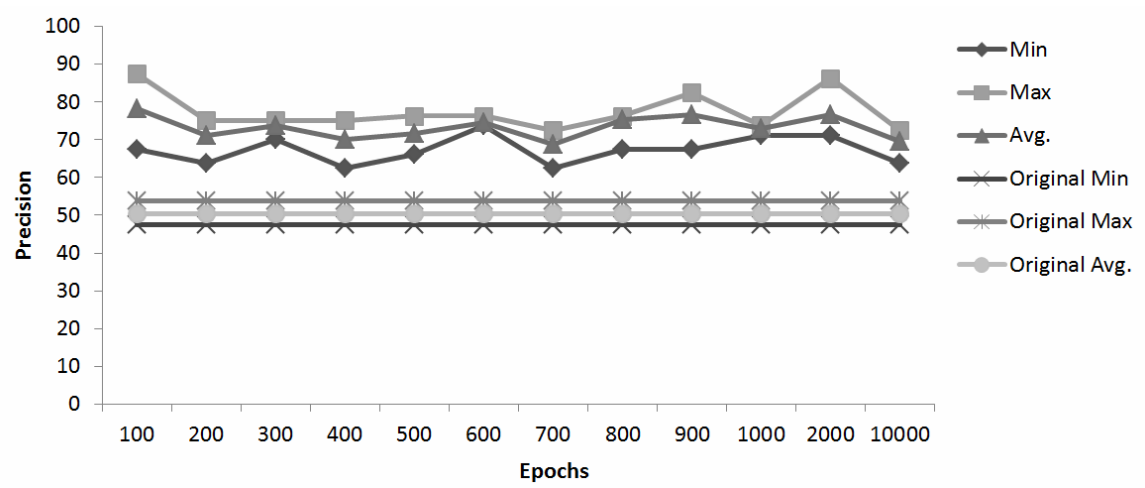

Fig. 4. The Min, Max, Average (Avg.) performance of the OMS and original version of the $K$-means over epochs $E=100,200 \ldots 1,000,2,000$, and 10,000

Table 1. Summarized performances of the OMS and the original version of the $K$-means in a database

\begin{tabular}{c|c|c|c}
\hline $\mathbf{E}$ & Min & Max & Average \\
\hline $\mathbf{1 0 0}$ & 67.50 & $\mathbf{8 7 . 5 0}$ & $\mathbf{7 8 . 3 3}$ \\
\hline $\mathbf{2 0 0}$ & 63.75 & 75.00 & 71.25 \\
\hline $\mathbf{3 0 0}$ & 70.00 & 75.00 & 73.75 \\
\hline $\mathbf{4 0 0}$ & 62.5 & 75.00 & 70.00 \\
\hline $\mathbf{5 0 0}$ & 66.25 & 76.25 & 71.67 \\
\hline $\mathbf{6 0 0}$ & 73.75 & 76.25 & 74.58 \\
\hline $\mathbf{7 0 0}$ & 62.50 & 72.50 & 68.75 \\
\hline $\mathbf{8 0 0}$ & 67.50 & 76.25 & 75.42 \\
\hline $\mathbf{9 0 0}$ & 67.50 & 82.50 & $\mathbf{7 6 . 6 7}$ \\
\hline $\mathbf{1 , 0 0 0}$ & 71.25 & 73.75 & 72.92 \\
\hline $\mathbf{2 , 0 0 0}$ & 71.25 & $\mathbf{8 6 . 2 5}$ & 76.67 \\
\hline $\mathbf{1 0 , 0 0 0}$ & 63.75 & 72.50 & 69.58 \\
\hline Average & $\mathbf{6 7 . 2 9}$ & $\mathbf{7 7 . 4 0}$ & $\mathbf{7 3 . 3 0}$ \\
\hline Original & 47.50 & $\mathbf{5 3 . 7 5}$ & $\mathbf{5 0 . 4 2}$ \\
\hline
\end{tabular}

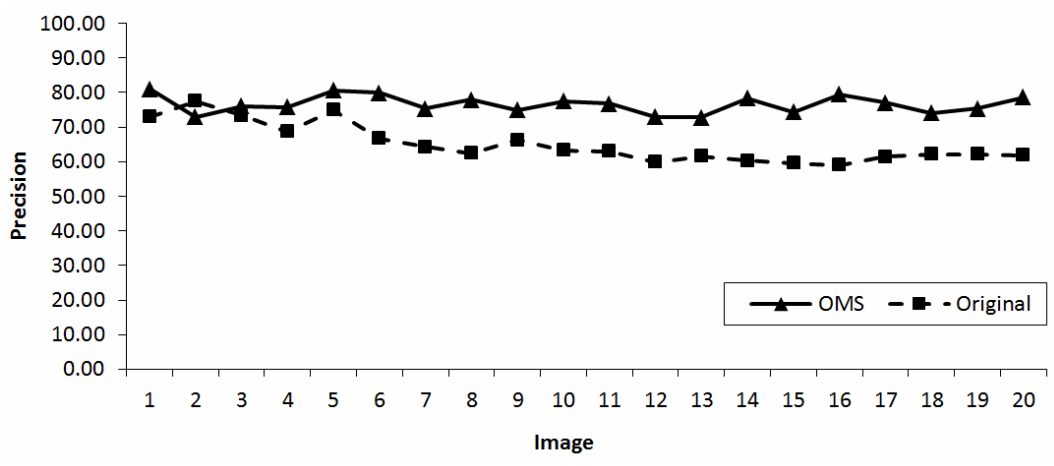

Fig. 5. The curve of the average precision over experimented queries. The solid line marked by triangles presents the trend of performance of our proposed Orientation Matching Scheme (OMS). The dotted line marked by squares presents the trend of performance of the original Gabor feature method 
is clear from the curve that the difference in performance between the OMS and the original method is significant.

\section{Conclusions}

In this paper, we have suggested an Orientation Matching Scheme (OMS) for texture information comparison. Compared to the conventional method, the experimental results show that our proposed OMS can really improve the performance of recognizing rotated texture patterns, which is a very popular fact. Although we choose the Gabor filters feature to present the work for the sake of convenience, the OMS is believed to work nicely with all multi-orientation feature-extracting approaches. Besides, the $K$-means algorithm is purposely chosen to illustrate the performance of the OMS model because this method will process a lot of comparisons during the clustering progress. By that way, we can ensure the good characteristics, as well as the high performance, of the OMS. Moreover, the OMS has the ability to extend the scope for scale matching as well. So our future work will focus on extending the OMS and applying it to the scale matching problem.

\section{REFERENCES}

[1] R. M. Haralick, "Statistical and structural approaches to texture," Proceedings of the IEEE, Vol.67, 1979, pp.786-804.

[2] G. R. Cross and A. K. Jain, "Markov Random Field Texture Models," Pattern Analysis and Machine Intelligence, IEEE Transactions on, Vol.PAMI-5, 1983, pp.25-39.

[3] A. R. Backes and O. M. Bruno, "A New Approach to Estimate Fractal Dimension of Texture Images," presented at the Proceedings of the 3rd international conference on Image and Signal Processing, Cherbourg-Octeville, France, 2008.

[4] A. K. Jain and F. Farrokhnia, "Unsupervised texture segmentation using Gabor filters," in Systems, Man and Cybernetics, 1990. Conference Proceedings., IEEE International Conference on, 1990, pp.14-19.

[5] R. Azencott, et al., "Texture classification using windowed Fourier filters," Pattern Analysis and Machine Intelligence, IEEE Transactions on, Vol.19, 1997, pp.148-153.

[6] A. R. Backes, et al., "Texture analysis using graphs generated by deterministic partially self-avoiding walks," Pattern Recogn., Vol.44, 2011, pp.1684-1689.

[7] M. Varma and R. Garg, "Locally Invariant Fractal Features for Statistical Texture Classification," in Computer Vision, 2007. ICCV 2007. IEEE 11th International Conference on, 2007, pp.1-8.

[8] J. Han and K.-K. Ma, "Rotation-invariant and scale-invariant Gabor features for texture image retrieval," Image Vision Comput., Vol.25, 2007, pp.1474-1481.

[9] S. A. Beck J, Ivry R. , "Spatial frequency channels and perceptual grouping in texture segregation," Computer Vision, Graphics, and Image Processing, Vol.37, 1987, pp.299-325.

[10] J. G. Daugman, "Complete discrete 2-D Gabor transforms by neural networks for image analysis and compression," Acoustics, Speech and Signal Processing, IEEE Transactions on, Vol.36, 1988, pp.1169-1179.

[11] W. Y. Ma and B. S. Manjunath, "Texture features and learning similarity," in Computer Vision and Pattern Recognition, 1996. Proceedings CVPR '96, 1996 IEEE Computer Society Conference on, 1996, pp.425-430. 


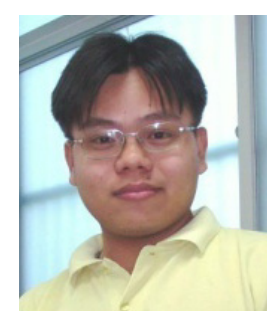

\section{Nguyen Cao Truong Hai}

He received a BS degree in Computer Science from the University of Sciences in Ho Chi Minh, Vietnam and an MS degree in Computer Engineering from Chonnam National University in 2006 and 2009, respectively. He is currently undertaking a doctorate course as a member of the Information Retrieval Lab at Chonnam University. His research interests include text classification; content- based image search, object recognition, nlp, and data mining.

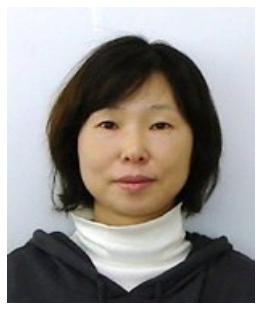

\section{Do-Yeon Kim}

She received an MS degree from Dongguk University. She is now undertaking a doctorate degree course as a member of the Information Retrieval Lab at Chonnam National University. Her research interests are in the areas of information retrieval, image retrieval, ontology, and the semantic web.

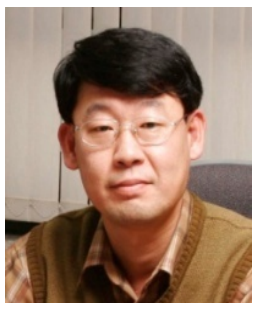

\section{Hyuk-Ro Park}

He received a BS degree from Seoul National University; and his MS and Ph.D. degrees from KAIST. He has been a professor at Chonnam National University since 1999. He is now in charge of the Information Retrieval Lab at Chonnam University. His research interests are in the areas of information retrieval, the Q\&A system, natural language processing, database system, and data mining. 